\title{
Solving the Multi-Objective Travelling Salesman Problem with Real Data Application
}

\author{
Barraq Subhi Kaml* and Mohamed Saad Ibrahim ${ }^{* *}$ \\ Ministry of Higher Education and Scientific Research, Baghdad-Iraq. \\ Corresponding Auther: barraq_1978@ukr.net* , Blackhawk_is@yahoo.com**
}

\begin{abstract}
The aim of this paper is building a mathematical model for Travelling salesman problem (TSP) with multi-objective; the model describes the problem of (TSP) with three objectives (cost, distance, time), Real data were collected with a sample of twenty states of United State of America, Three methods were used (Branch and Bound algorithm, Nearest neighbor and two-way exchange improvement heuristic), The comparison was conducted among results reached.

To solve the problem multi-objective of (TSP), The weighted model demonstrated the effectiveness and flexibility to solve real problems of multi-objective (TSP), where it can be said that it is impossible to solve this problem without resorting to multiple -objective mathematical models, In other words, the number of possible rout for the 20 town is $\{(n-1) !=19 !=121645100408832000\}$, to find the optimal routs among these routs it takes very long time and a lot of effort, here stand out importance of two-way exchange improvement heuristic algorithm, where this rout is satisfactory to the decision maker in terms of cost, distance and time.

[DOI: $10.22401 / \mathrm{JNUS} .21 .3 .18]$
\end{abstract}

Keywords: Traveling Salesman Problem (TSP), mathematical programming formulation, multiobjective model, weighted-sum method, Branch \& Bound algorithm, nearest neighbor, two-way exchange improvement heuristic

\section{1-Introduction}

This paper has focused attention of study traveling salesman problem (TSP) when there are multi-objective, as this problem is one of the problems of the combinatorial optimization which has gained widespread reputation and interest from researchers so as to simple formulation and its important applications, This concern came from the actual need of many productive sectors and companies that distribute products locally or imported to customers or other industrial sectors.

The Traveling Salesman Problem (TSP) is the problem of finding minimum expensive to visit a set of cities, a particular sequence, beginning and the end at the same city, each city must be visited exactly one time. Since this problem was formulated mathematically, the essence of the problem was in the area of combinatorial optimization. There is an important difference can be made between the symmetric TSP and the asymmetric TSP, for the symmetric case all distances are equal $\{d i j=d j i\}$ no matter what it was if we travel from city $(i)$ to city $(j)$ or on the contrary because the distance is the same, in the second case the distances are not equal for all pairs of cities. This kind of problems arises when we do not transact with locative distances between cities but with the time and cost associated with travelling between locations.

\section{2-Historical overview:}

The problem (TSP) was first mentioned by German scientist Karl Menger in the book "The Successful Rover" in 1832. He was the first scientist wrote in this problem, where he wanted to find $\{l(\mathrm{c})\}$, where:

$$
l(\mathrm{c})=\sup \sum_{i=1}^{n-1} \operatorname{dist}\left(\mathrm{x}_{i}, \mathrm{x}_{i+1}\right)
$$

Where sup (supremum) refer to the highest values, which is take it on every selection $\left\{x_{1}, x_{2}, \ldots, x_{n-1}\right\}$,on $C$, In the order placed by $C$, Karl Menger has to solve this problem is that can be examine all the final set $X$ for $C$ that is:

$$
\{\exists n \in N: X \subset C,|X|=n\}
$$


Then we take the minimum value for all ranks $X$, therefore, define each set $X$ for metric space $\{S: \lambda(X)\}$ it is the length of the shortest path through which it passes, and it has proved the following:

$$
l(\mathrm{c})=\sup _{X} \lambda(X)
$$

In 1930 Karl Menger presented the problem more clearly and considered it as a separate problem, in the same year winter put the problem under the name (travelling salesman), in the period between 1950 and 1960, the problem of the traveling vendor began to spread in the scientific community, especially in Europe and the United States of America.

In the meantime, when the challenge among the pioneers of algorithms increased, several researchers, including Dantzic and Johnson), succeeded in linear programming method to development method of cutting plane, In this new method, it was possible to solve the problem and find a tour among 49 cities, and proved lack of a shorter trip.

In subsequent decades the problem was studied by many mathematicians, physicists, chemists and other scientists.

In 1972, Richard M. Karp indicated that the Hamiltonian cycle problem was NPcomplete, which means implicitly the NPcruelty of TSP. This has provided an explanation mathematically for arithmetic difficulty in finding the optimal tours. It is then scientists have since developed many methods to solve the problem directly, such as genetic algorithms and mixed linear programming. The possible forms of the problem (TSP) are as follows:

\section{2-1- Single versus multiple warehouses}

In the case of a single warehouse, all sellers start from and finish their tours at one point, on the other hand, if there are multiple warehouse with a number of sellers present in each warehouse, sellers can either return to the original warehouse after completing their tour or return to any warehouse with a restriction that the initial number of sellers in each warehouse is still the same after each travel, The first case is called "fixed destination case", and the second "non-fixed destination case".

\section{2-2- Fixed charges}

When the number of sellers is usually constant, each of them has a fixed cost incurred each time the seller is used in the solution. In this case, reducing the number of them that has been activated in the solution may also be a source of interest.

\section{2-3- Time restriction}

In this type, some cities need to visit at certain time intervals, this is great protraction of the multiple traveling salesman problem with time, because to have commonly used applications in the real life such as in school bus, and airline scheduling problems[3].

\section{3-Formulating the Travelling Salesman Problem (TSP)}

When $i=j, x_{i j}$ does not exist so it is not included in the model. We now give the mathematical programming formulation of the asymmetric TSP [6].

$$
\text { Minimize: } \sum_{i=1}^{n} \sum_{j=1}^{n} c_{i j} x_{i j}
$$

\section{(Minimizetotal cost of tour)}

SubjectTo: $\sum_{j=1}^{n} x_{i j}=1 ; \quad i=1, \ldots, n$

(Leaveeach cityexactlyonce)

$$
\sum_{i=1}^{n} x_{i j}=1 ; j=1, \ldots, n
$$

(Visiteachcityexactlyonce)

$$
\begin{aligned}
& x_{i j} \in\{0,1\} ; i, j=1, \ldots, n \\
& \left(x_{i j} \text { is abinarydecision varible }\right) \\
& \left\{(i, j): x_{i j}=1, i, j=2, \ldots, n\right\} \\
& \sum_{i, j \in S}^{n} x_{i j}=|S|-1 ; S \subseteq\{2, \ldots, n\}, 2 \leq|S| \leq n-1
\end{aligned}
$$

(Subtour elimination).

\section{4-Methods of solving a TSP}

In literatures there are many different ways to solve TSP, efficiency techniques and also results. Let us refer to a summary of the most widely used methods:

\section{4-1- Branch and Bound algorithm}

The B And B algorithm starts with the optimal solution associated with the allocation 
problem, If the solution represents a path, the process ends, otherwise we impose constraints to remove the sub-tour, this can create as many different branches as variables associated with one of the sub-tour, each branch represents put one of the variables for the sub-tour equal to zero [1]. Initially before solving the problem, that we specify the upper bound select any rout connected (that does not contain sub-tour), and preferably use intuitions because it produce a higher limit than any rout, then we solve the problem as a normal allocation problem If the solution represents a Hamiltonian cycle (that does not contain sub-tour), the solution will stop and we consider the resulting solution is the optimal solution, If the solution to the problem of allocation does not represent Hamiltonian cycle, we'll assign the resulting solution as a lower bound, and that any solution that produces greater or equal to the upper bound path will ignore. Then select one of the sub routs to branch it preferably the selected sub rout contains the minimum number of cities (node), because it creates fewer braches. Note that the basic idea of branching to smashing one of the sub routs and modifying organic variables for the other subrout automatically.

\section{4-2 Nearest neighbor}

Intuitive methods are defined as a guessing state for the priority of choosing a point for another within the solution for some objectives often intuitions can find good solutions to the problem but they may not be optimal solutions. A good solution can be found to the problem of a traveling salesman by starting from the city the specific node, and then connecting it with the nearest city that has not been visited before, and continues the process until the Hamiltonian cycle is formed [4].

1. Choose the city randomly.

2. Find the node closest to it and non-visited.

3 . Is there a node that has not been visited? If the answer is yes, repeat step 2 .

4. We return to the city from which we started.

Thus we get the Hamiltonian cycle with $\left\{O\left(n^{2}\right)\right\}$, this method is useful and highly efficient because there is only one path to be formed, but it may not reach to the objective well.

\section{4-3 Two-way exchange improvement heuristic}

This method is also called Two-optimal improvement heuristic; the basic principle of this method is to modify the solution to a better solution. By modifying the tour, two arcs are deleted and reconnected the paths in a different way which reduces the total distance between nodes of the network until no deleted pair of arcs is found [8].

\section{5-The Main Features of Decision Making of Multi-Objective}

It can be said that the decision-maker actually seeks to achieve several objective; therefore the traditional model (one- objective) is no longer appropriate for him. The traditional framework for analyzing decisionmaking, presumably assume that there are three elements, Decision maker (personal or organization defined as a single entity), a set of available choices, and finally specific criteria (objective). Specific criteria are used to associate them with a number of alternatives so it can be arranged in the form of a set to get the optimal value that can be achieved from the selected objectives, Decision makers often do not mind to organize a set of possible solutions that are subject to one (objective) criterion but prefer the presence of a centrist compromise solution involves several objectives[2].

\section{6-Definition of efficient solution}

A solution $\left\{x^{1} \in X\right\}$ is called efficient if and only if there is no other solution $\{x \in X\}$ where

$$
\left\{f_{k}(x) \geq f_{k}\left(x^{1}\right) \forall k(k=1,2, \ldots, p)\right\} \text {. }
$$

The inequality being strict for at least one $\left\{k\left(f_{k}(x)>f_{k}\left(x^{1}\right)\right)\right\}$. Each solution $x$ has a point $\left\{F=\left(f_{1}(x), \ldots, f_{p}(x)\right)\right\}$ as representation in the objective function space. 


\section{7- Optimizing a Weighted-Sum of the Objective Functions}

The process of computation of (efficient/ non-dominated) solutions more utilized consists in solving a scalar problem in which the objective function is a weighted-sum of the $p$ original objective functions with positive weights $\omega_{k}$ :

minimize (or maximize) $F_{W}=$ $\sum_{k=1}^{p} \omega_{k} f_{k}(\mathrm{x})$

SubjectTo:

$$
x \in X
$$

if $x^{1} \in X$ is a solution to the problem $\min _{x \in X} \sum_{k=1}^{p} \omega_{k} f_{k}(\mathrm{x})$ for $W=\left(\omega_{1}, \ldots, \omega_{p}\right)$, where $\omega_{k}>0, k=1, \ldots, p$, and $\sum_{k=1}^{p} \omega_{k}=1$, then $x^{1}$ is an efficient solution to the multi-objective problem. The truthfulness of this proposition can be shown as follows. Suppose that $x^{1}$ is not efficient. Then, there is an $x^{2} \in X$ such that $f_{k}\left(x^{2}\right) \geq f_{k}\left(x^{1}\right), k=1, \ldots, p$, and the inequality is strict for at least one $k$. But $x^{1}$ was obtained by optimizing a weightedsum objective function with strictly positive weights then $\sum_{k=1}^{p} \omega_{k} f_{k}\left(x^{2}\right)>\sum_{k=1}^{p} \omega_{k} f_{k}\left(x^{1}\right)$, which contradicts the hypothesis that $x^{1}$ minimizes the weighted-sum objective function [7].

\section{8-Data type}

Before building the mathematical model of the problem, we must identify our data and statement qualitatively; therefore we will define the model data type, the data related to the problem are concerned with objectives placed by the decision maker and these objectives are defined according to the following indicators:

- Choose the route that achieves the least time it takes to reach between any two cities in the tour; this objective is expressed by indicator (time).

- Choose the shortest route possible connecting between any two cities in the tour; this objective is expressed by indicator (distance).

- Choose the route that achieves the lowest cost to reach between any two cities in the tour; this objective is expressed by indicator (cost). Data problem (cost, distance, and time) was obtained by the web sites [9]. 


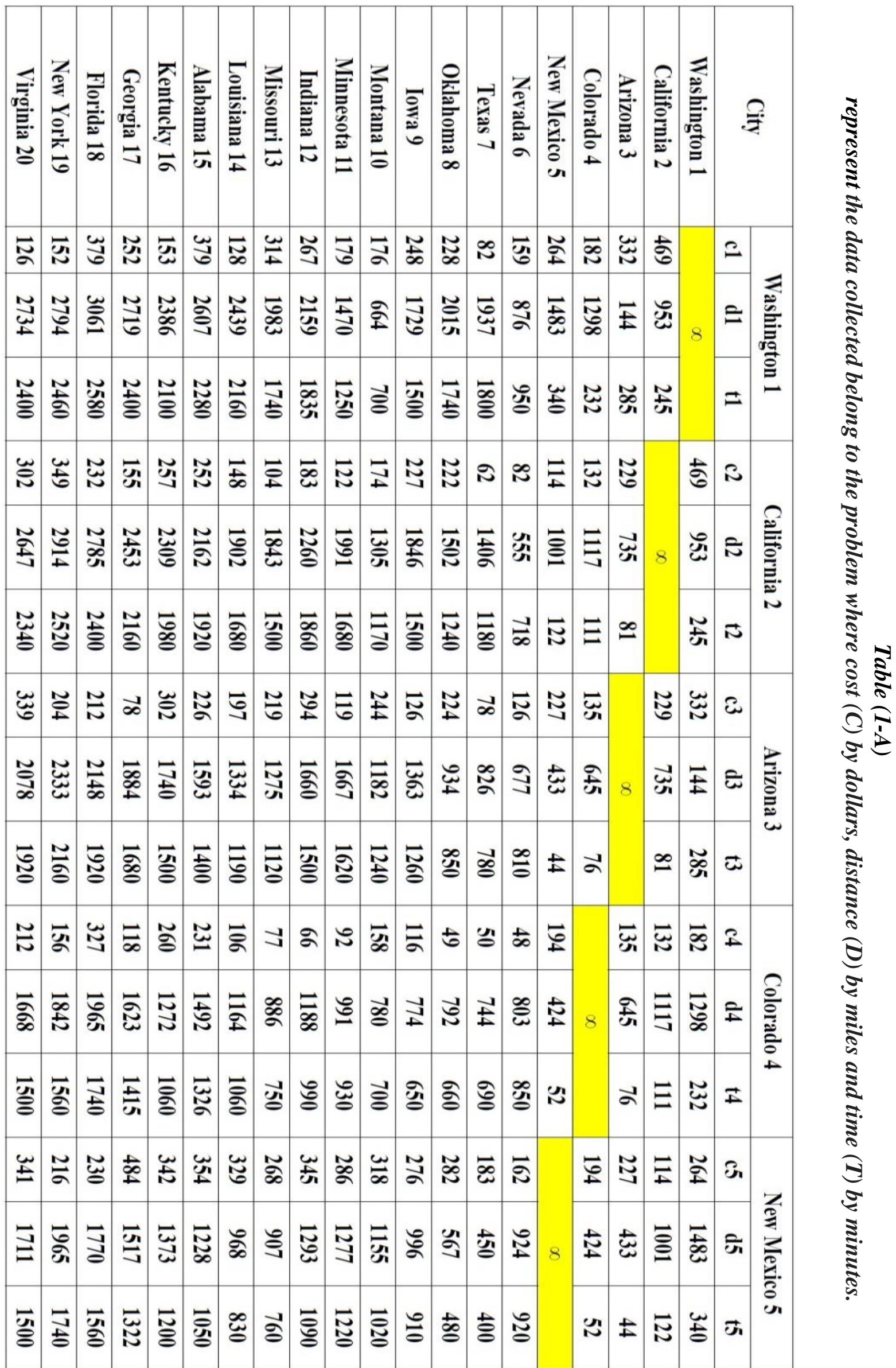




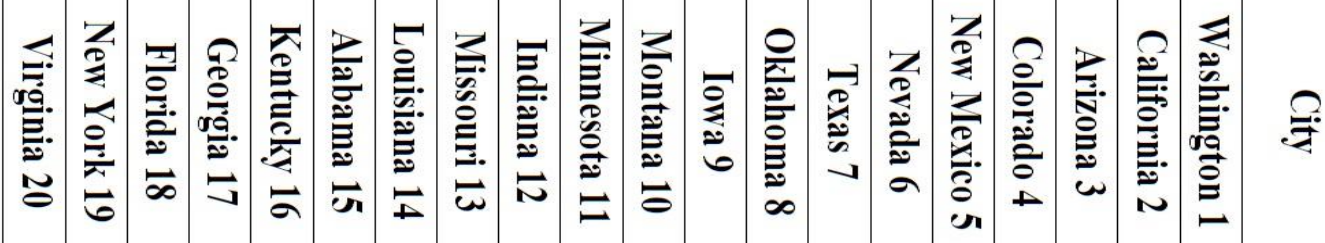

స્ळ

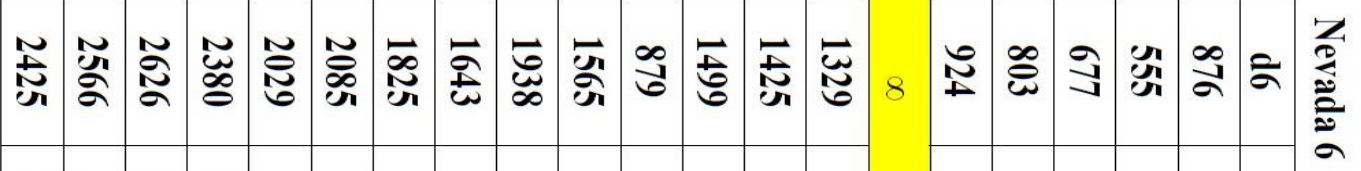

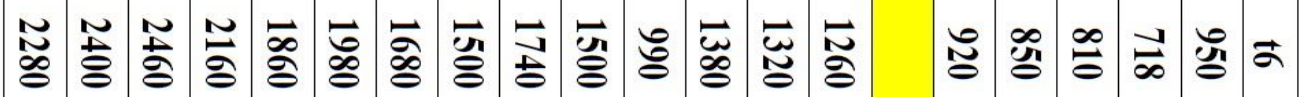

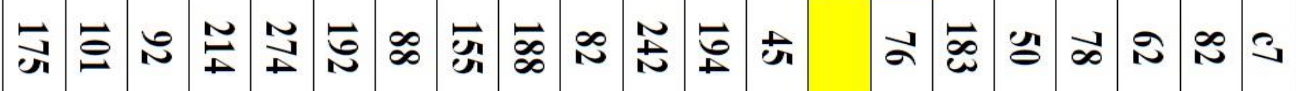

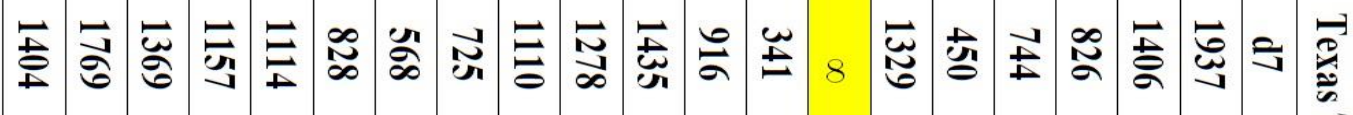

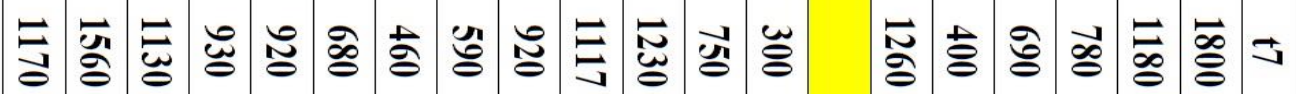

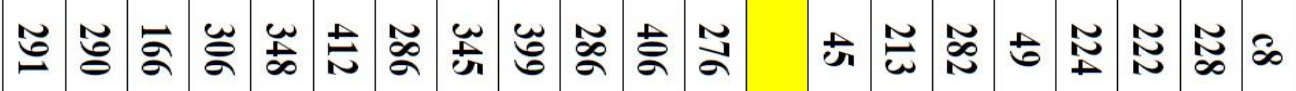

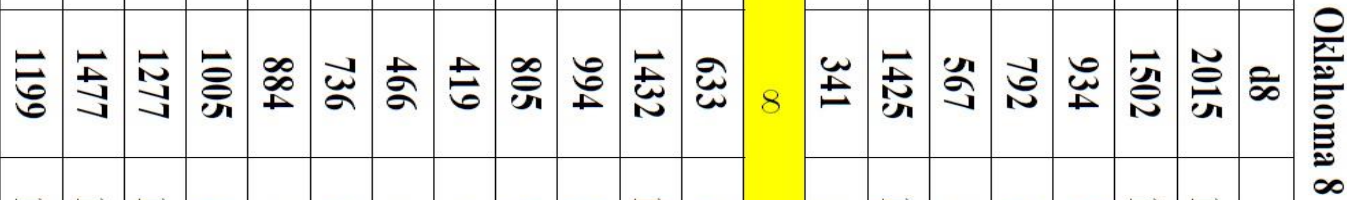

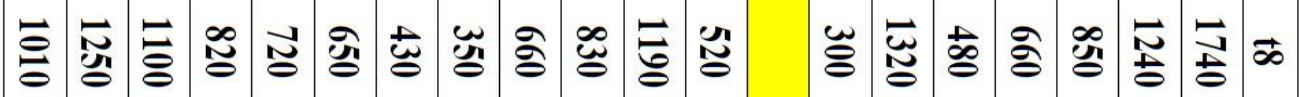

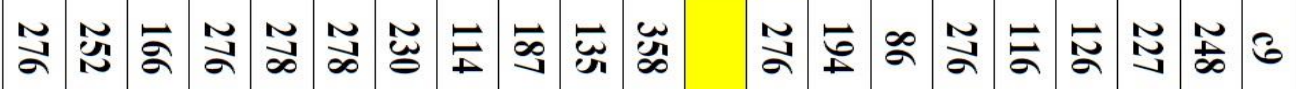
总总岕总

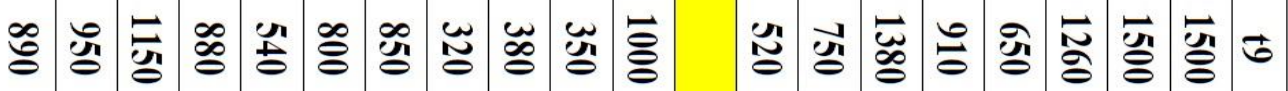

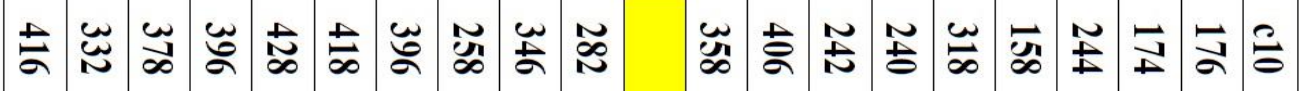
芯昘苔

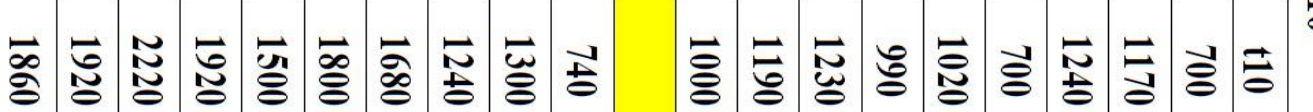




\begin{tabular}{|c|c|c|c|c|c|c|c|c|c|c|c|c|c|c|c|c|c|c|c|}
\hline 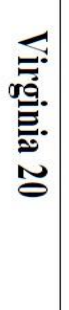 & 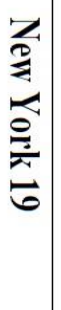 & 걸 & 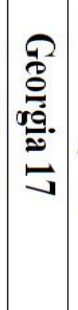 & 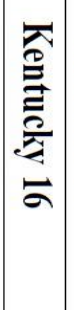 & 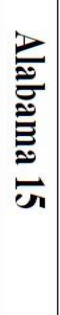 & 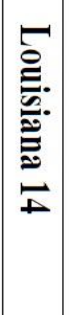 & 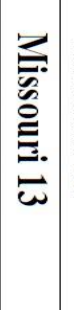 & 言 & 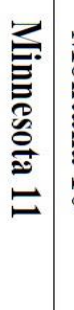 & 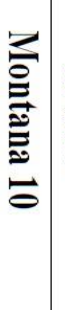 & 包 & 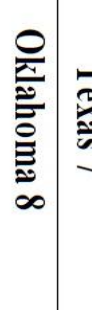 & 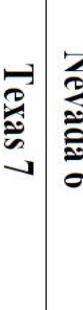 & 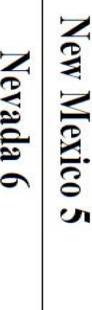 & 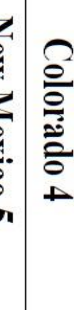 & 芯 & 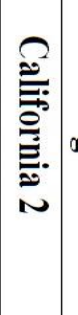 & 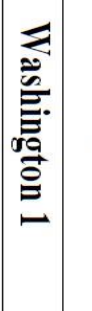 & $\varsubsetneqq$ \\
\hline$\widetilde{J}$ & $\vec{\Phi}$ & $\breve{\infty}_{\infty}$ & $\mathbb{N}$ & 岁 & 鸟 & 鸟 & $\stackrel{\infty}{-\infty}$ & $\tilde{a}$ & & 芯 & 岕 & $\tilde{\mathscr{E}}:$ & $\mathbb{S}$ & $\tilde{c} \tilde{\mathscr{a}}$ & i & $\vec{\sigma}$ & $\mathbb{N}$ & & \multirow{3}{*}{$=$} \\
\hline 矛 & $\overrightarrow{\widetilde{\varpi}}$ & $\vec{\Phi}$ & 声 & 曾 & 㥕 & 氖 & $\underset{\mathbf{N}}{\mathbf{N}}$ & 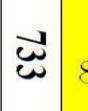 & 8 & : & 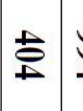 & $\stackrel{5}{ \pm}$ & స్త్ర & $\overline{\mathrm{Un}}$ & 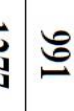 & $\overrightarrow{5}$ & 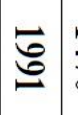 & $\stackrel{\mathrm{E}}{\mathrm{d}}$ & \\
\hline $\overrightarrow{\vec{\sigma}_{0}}$ & $\overrightarrow{\vec{\Phi}}$ & 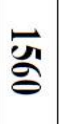 & $\overrightarrow{\mathrm{g}}$ & $\stackrel{\infty}{\Xi}$ & $\Xi$ & 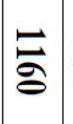 & $\hat{\partial}$ & 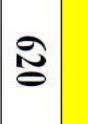 & & $\vec{E}$ & 怨 & 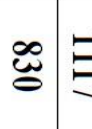 & 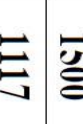 & 药 & $\ddot{\mathscr{\sigma}}$ & 竎 & $\overrightarrow{\widehat{a}}$ & 苔 & \\
\hline $\mathbb{N}$ & $\vec{N}$ & $\approx$ & $\overrightarrow{0}$ & 嵓 & 总 & $\overrightarrow{\mathrm{N}}$ & $\vec{\Xi}$ & & ت্ভ & 崩 & $\vec{\infty}$ & $\underset{\text { w }}{w}$ q & $\mathscr{\infty}$ & $\tilde{\Sigma}$ & 2 & $\stackrel{\mathscr{E}}{ \pm}$ & $\vec{\infty}$ & $\tilde{a}$ & \multirow{3}{*}{ : } \\
\hline ஜ̈ & $\overleftrightarrow{\infty}$ & 亏్తి & 工 & 告 & 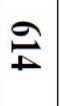 & $\underset{8}{\not}$ & 晜 & 8 & $\vec{\omega}$ & $\underset{\mathscr{\infty}}{\vec{\Phi}}$ & 壱 & 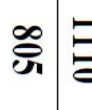 & 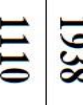 & 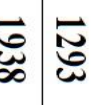 & $\vec{\infty}$ & $\bar{g}$ & 芯 & 药 & \\
\hline$\stackrel{\mathscr{C}}{\mathscr{C}}$ & Фे & $\stackrel{\infty}{\dot{d}}$ & 窎 & $\tilde{\Xi}$ & 岂 & $\vec{g}$ & $\underset{\mathscr{\circlearrowleft}}{\mathscr{\Xi}}$ & & $\bar{\Xi}$ & $\overrightarrow{\breve{\Xi}}$ & $\ddot{\ddot{g}}$ & $\widehat{\partial}$ & 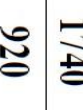 & $\vec{E}$ & 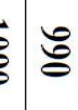 & 离 & $\overrightarrow{\stackrel{\infty}{g}}$ & 总 & \\
\hline$\underset{\infty}{\mathbb{\infty}}$ & $\widetilde{\Xi}$ & $\sigma$ & $\overrightarrow{\mathrm{g}}$ & 苔 & 忿 & $\vec{b}$ & & 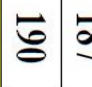 & $\vec{\infty}$ & 䓵 & $\overrightarrow{ \pm}$ & 志离 & 系 & $\tilde{\omega}$ & : & $\widetilde{\widetilde{\sigma}}$ & $\vec{\nexists}$ & $\ddot{\omega} \Xi$ & \multirow{3}{*}{$\bar{\vdots}$} \\
\hline$\stackrel{\circ}{\circ}$ & 心 & Б્ડ & $\mathbb{P}$ & $\overrightarrow{\mathrm{d}}$ & 今े & 岁 & 8 & $\ddot{\mathscr{\sigma}}$ & $\overrightarrow{\mathbf{N}}$ & 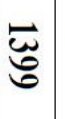 & 䜣 & $t$ & 岕 & 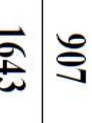 & 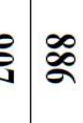 & 壱 & $\mid \begin{array}{l}\vec{\infty} \\
\dot{\omega}\end{array}$ & 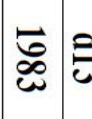 & \\
\hline$\vec{E}$ & $\stackrel{\bullet}{\varrho}$ & ஃ & gे & $\ddot{\ddot{g}}$ & 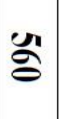 & 㐔 & & $\underset{\Xi}{\mathbb{O}}$ & & 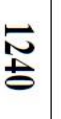 & $\underset{\mathbb{E}}{\mathbb{E}}$ & 惢 & 岁 & $\overrightarrow{\mathrm{E}}$ & 경 & 壱 & 䆧 & $\vec{\Xi}$ & \\
\hline $\mathbb{\mathfrak { N }}$ & $\vec{\Phi}$ & $\vec{I}$ & $\infty$ & $\begin{array}{l}n \\
\text { 去 } \\
\text {. }\end{array}$ & స్థ & & : & 讨 & $\overline{a ̆}$ & 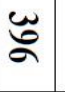 & 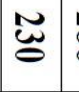 & $\underset{\mathscr{\sigma}}{\mathscr{\circ}} \approx$ & $\infty \approx \bar{\varnothing}$ & $\begin{array}{l}\bar{\infty} \\
\bar{\sigma}\end{array}$ & : & $\vec{v}$ & $\stackrel{F}{\infty}$ & $\mathbb{\infty}$ & \multirow{3}{*}{ 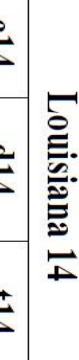 } \\
\hline $\overrightarrow{\vec{E}}$ & $\overrightarrow{\mathscr{\mathscr { c }}}$ & $\ddot{ٌ}$ & 岕 & 总 & $\begin{array}{l}\mathscr{\infty} \\
\infty\end{array}$ & 8 & 台 & 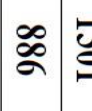 & $\stackrel{\vec{\leftrightarrows}}{\Xi}$ & 总 & 足 & 索 & שֶ. & 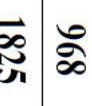 & $\vec{\nabla}$ & $\underset{ \pm}{\breve{\Delta}}$ & 总 & 䓫 & \\
\hline$\dot{d}$ & 芯 & $\widehat{\mathrm{g}}$ & $\hat{\mathrm{g}}$ & $\vec{b}$ & $\vec{\Xi}$ & & & $\vec{g}$ & $\vec{\Xi}$ & $\overrightarrow{\widehat{\partial}}$ & 荈 & $\stackrel{\overrightarrow{\breve{c}}}{\stackrel{t}{g}}$ & ta & 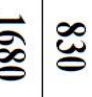 & $\stackrel{\Xi}{\mathrm{g}}$ & $\vec{\Xi}$ & $\overrightarrow{\ddot{\partial}}$ & $\stackrel{\widetilde{a}}{\bar{g}}$ & \\
\hline$\tilde{\Xi}$ & $\vec{\Xi}$ & $\overrightarrow{\sigma_{0}}$ & 悹 & $\widetilde{\swarrow}$ & & $\stackrel{\sim}{\infty}$ & 忍 & 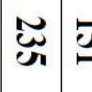 & 元 & $\vec{\infty}$ & $\mathbb{\infty}$ & 志 & $\underline{\underline{s}}$ & 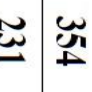 & $\tilde{\underline{u}}$ & N & 总 & $\underset{J}{\sigma}$ & \multirow{3}{*}{ 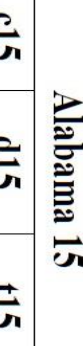 } \\
\hline $\overrightarrow{\mathrm{\partial}}$ & 心ิ & 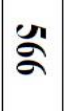 & $\vec{\exists}$ & 岁 & 8 & $\mid \begin{array}{c}\ddot{\mathscr{\infty}} \\
\ddot{\infty}\end{array}$ & 今े & $\stackrel{2}{ \pm}$ & 㝕 & 芯 & 足 & 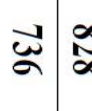 & $\underset{\widetilde{\infty}}{\infty}$ & 愛 & 䓠总 & 总 & 芯 & & \\
\hline 8 & $\stackrel{\alpha}{\leftrightarrows}$ & & $\tilde{\Xi}$ & 志 & & $\vec{d}$ & 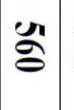 & 告 & $\Xi$ & 8 & $\stackrel{\circ}{0}$ & 의 & 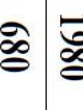 & 常 & $\begin{array}{l}5 \\
5\end{array}$ & $\overrightarrow{\vec{\Xi}}$ & & & \\
\hline
\end{tabular}




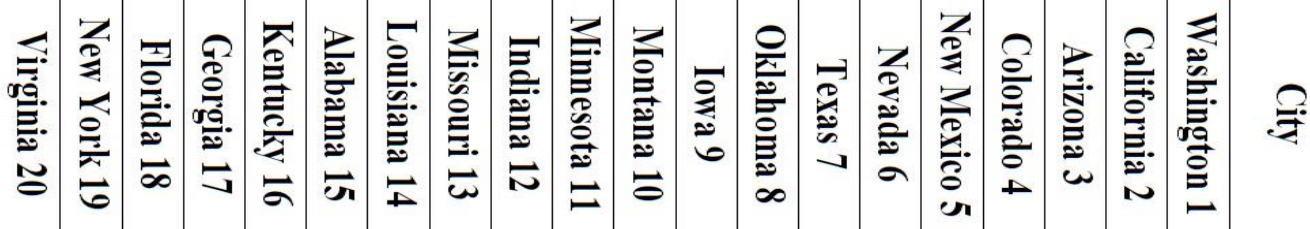

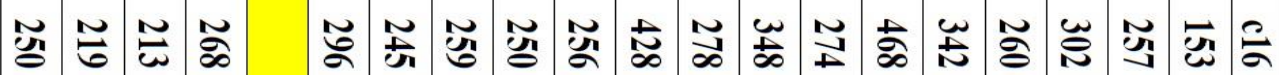

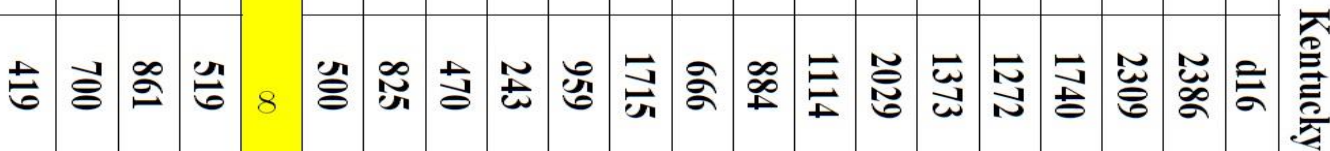

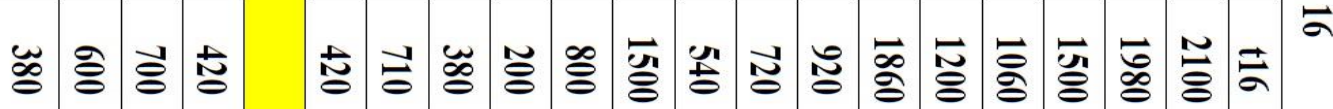

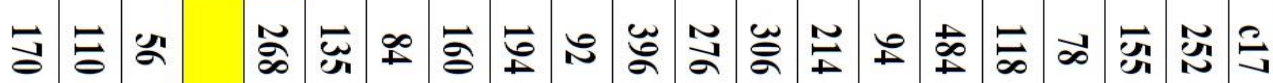

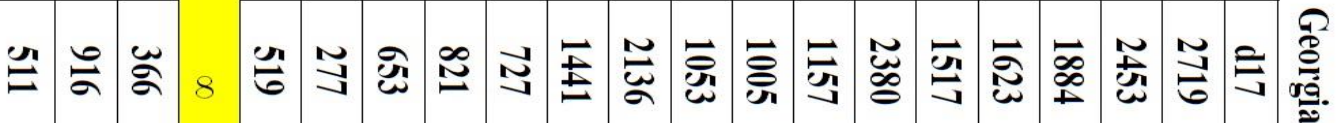

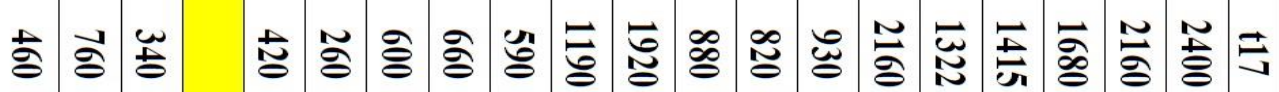

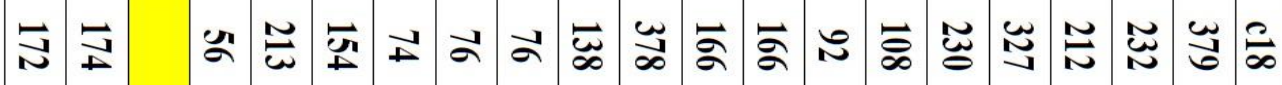

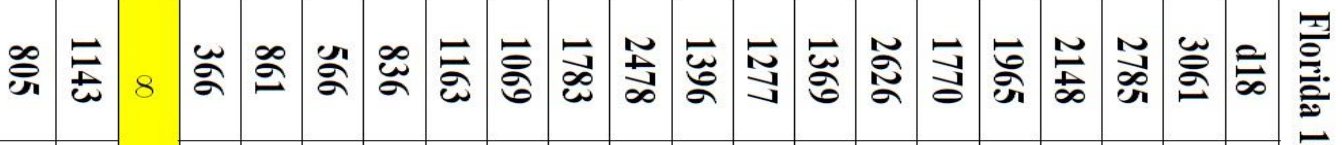

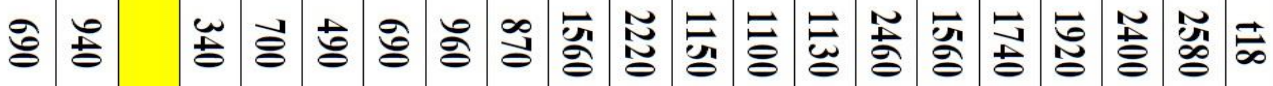

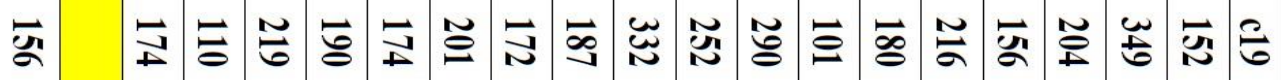

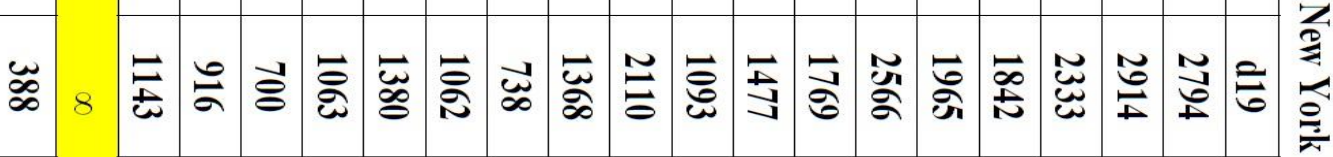

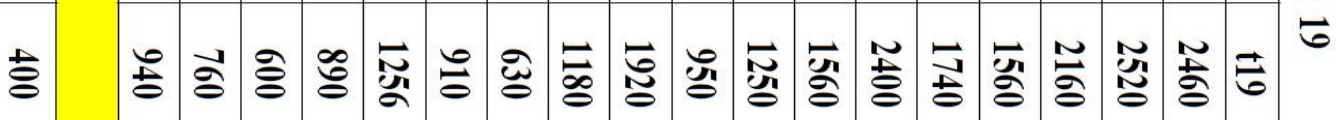

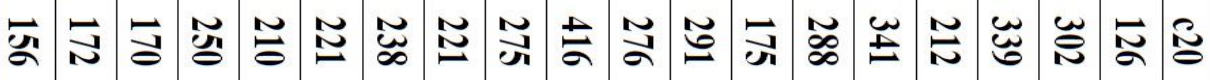

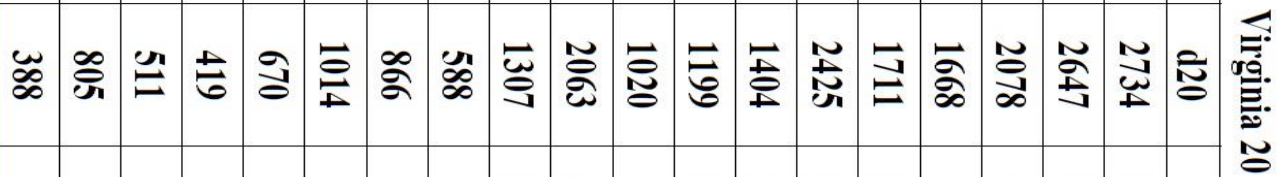

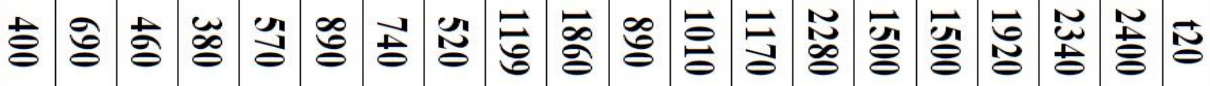




\section{9-The Practical Part}

A multialbibjective linear programming model will be built to solve the problem of a traveling sales man in the United States by formulating the (TSP) which is mentioned in paragraph (3) and using the data shown in the above tables, as follows:

\section{9-1 Decision Variables}

Let $x_{i j}$ represent the binary variable or in other words

$$
x_{i j}=\left\{\begin{array}{l}
1, \text { if the arc from } i \text { to } j \text { is selected } \\
\text { for all } i \neq j, i, j=1,2, \ldots, n \\
0, \quad \text { otherwise }
\end{array}\right.
$$

\section{9-2 Objective functions}

The three objective functions (cost, distance and time) $\left\{f_{1}, f_{2}, f_{3}\right\}$ are formulated respectively as follows:

Firstly, the objective function of achieving the maximum reduction of the total cost

$$
f_{1}(\mathrm{x}) \sum_{i=1}^{20} \sum_{j=1}^{20} c_{i j} x_{i j}
$$

Minimize $f_{1}(\mathrm{x})=469 x_{1,2}+332 x_{1,3}+182 x_{1,4}+246 x_{1,5}$ Totalcost

$$
\begin{aligned}
& +159 x_{1,6}+82 x_{1,7}+228 x_{1,8}+248 x_{1,9} \\
& +179 x_{1,11}+267 x_{1,12}+314 x_{1,13}+128 x_{1,14} \\
& +379 x_{1,15}+153 x_{1,16}+176 x_{1,10}+252 x_{1,17} \\
& +379 x_{1,18}+152 x_{1,19}+126 x_{1,20}+\ldots \\
& +126 x_{20,1}+302 x_{20,2}+339 x_{20,3}+212 x_{20,4} \\
& +341 x_{20,5}+288 x_{20,6}+175 x_{20,7}+291 x_{20,8} \\
& +276 x_{20,9}+416 x_{20,10}+275 x_{20,11}+221 x_{20,12} \\
& +238 x_{20,13}+221 x_{20,14}+210 x_{20,15}+250 x_{20,16} \\
& +170 x_{20,17}+172 x_{20,18}+156 x_{20,19}
\end{aligned}
$$

Secondly, the objective function of achieving the maximum reduction of the total distances

$$
f_{2}(\mathrm{x}) \sum_{i=1}^{20} \sum_{j=1}^{20} d_{i j} x_{i j}
$$

Minimize $f_{2}(\mathrm{x})=953 x_{1,2}+144 x_{1,3}+1298 x_{1,4}+1483 x_{1,5}$

$$
\begin{gathered}
+876 x_{1,6}+1937 x_{1,7}+1015 x_{1,8}+1729 x_{1,9} \\
+1470 x_{1,11}+2159 x_{1,12}+1983_{1,13}+2439 x_{1,14} \\
+2607 x_{1,15}+2386 x_{1,16}+664 x_{1,10}+2719 x_{1,17} \\
+3061 x_{1,18}+2794 x_{1,19}+2734 x_{1,20} \ldots+2734 x_{20,1} \\
+2647 x_{20,2}+2078 x_{20,3}+1668 x_{20,4}+1711 x_{20,5} \\
+2425 x_{20,6}+1404 x_{20,7}+1199 x_{20,8}+1020 x_{20,9} \\
+2063 x_{20,10}+1307 x_{20,11}+588 x_{20,12}+866 x_{20,13} \\
+1014 x_{20,14}+670 x_{20,15}+419 x_{20,16}+511 x_{20,17} \\
805 x_{20,18}+388 x_{20,19}
\end{gathered}
$$

Thirdly, the objective function of achieving the maximum reduction of the total time

$$
f_{3}(\mathrm{x}) \sum_{i=1}^{20} \sum_{j=1}^{20} t_{i j} x_{i j}
$$

Minimize $f_{3}(\mathrm{x})=245 x_{1,2}+285 x_{1,3}+232 x_{1,4}+340 x_{1,5}+950 x_{1,6}+1800 x_{1,7}+1740 x_{1,8}+1500 x_{1,9}+700 x_{1,10}$

$$
\begin{aligned}
& +1250 x_{1,11}+1835 x_{1,1,2}+1740_{1,13}+2160 x_{1,14}+2280 x_{1,15}+2100 x_{1,16}+2400 x_{1,17}+2580 x_{1,18} \\
& +2460 x_{1,19}+2400 x_{1,20} \ldots+2400 x_{20,1}+2340 x_{20,2}+1920 x_{20,3}+1500 x_{20,4}+1500 x_{20,5}+ \\
& 2280 x_{20,6}+1170 x_{20,7}+1010 x_{20,8}+890 x_{20,9}+1860 x_{20,10}+1199 x_{20,11}+520 x_{20,12}+740 x_{20,13}+ \\
& 890 x_{20,14}+570 x_{20,15}+380 x_{20,16}+460 x_{20,17}+690 x_{20,18}+400 x_{20,19}
\end{aligned}
$$

\section{SubjectTo:}

The constraints of the multi-objective problem can be represented in the following mathematical formula:

$$
\left.\begin{array}{c}
\sum_{j=1}^{20} x_{(i=1) j}=1, \sum_{j=1}^{20} x_{(i=2) j}=1, \sum_{j=1}^{20} x_{(i=3) j}=1, \\
\sum_{j=1}^{20} x_{(i=4) j}=1, \sum_{j=1}^{20} x_{(i=5) j}=1, \sum_{j=1}^{20} x_{(i=6) j}=1, \\
\sum_{j=1}^{20} x_{(i=7) j}=1, \sum_{j=1}^{20} x_{(i=8) j}=1, \sum_{j=1}^{20} x_{(i=9) j}=1, \\
\sum_{j=1}^{20} x_{(i=10) j}=1, \sum_{j=1}^{20} x_{(i=11) j}=1, \sum_{j=1}^{20} x_{(i=12) j}=1,
\end{array}\right\}
$$




$$
\left.\begin{array}{l}
\sum_{j=1}^{20} x_{(i=13) j}=1, \sum_{j=1}^{20} x_{(i=14) j}=1, \sum_{j=1}^{20} x_{(i=15) j}=1, \\
\sum_{j=1}^{20} x_{(i=16) j}=1, \sum_{j=1}^{20} x_{(i=17) j}=1, \sum_{j=1}^{20} x_{(i=18) j}=1, \\
\sum_{j=1}^{20} x_{(i=19) j}=1, \sum_{j=1}^{20} x_{(i=20) j}=1 . \\
\sum_{i=1}^{20} x_{i(j=1)}=1, \sum_{i=1}^{20} x_{i(j=2)}=1, \\
\sum_{i=1}^{20} x_{i(j=3)}=1, \sum_{i=1}^{20} x_{i(j=4)}=1, \\
\sum_{i=1}^{20} x_{i(j=5)}=1, \sum_{i=1}^{20} x_{i(j=6)}=1, \\
\sum_{i=1}^{20} x_{i(j=7)}=1, \sum_{i=1}^{20} x_{i(j=8)}=1, \\
\sum_{i=1}^{20} x_{i(j=9)}=1, \sum_{i=1}^{20} x_{i(j=10)}=1, \\
\sum_{i=1}^{20} x_{i(j=11)}=1, \sum_{i=1}^{20} x_{i(j=12)}=1, \\
\sum_{i=1}^{20} x_{i(j=13)}=1, \sum_{i=1}^{20} x_{i(j=14)}=1, \\
\sum_{i=1}^{20} x_{i(j=15)}=1, \sum_{i=1}^{20} x_{i(j=16)}=1, \\
x_{i j}^{20} x_{i(j=17)}=1, \sum_{i=1}^{20} x_{i(j=18)}=1, \sum_{j=1}^{20} x_{i(j=20)}=1 .
\end{array}\right\}
$$

The above model is a problem of achieving optimization of multi-objective, when we solved it, the results of this model includes a conflict among three objectives and cannot achieve the maximum reduction of objective simultaneously without increasing one of the objectives, Therefore, the method of weightssum was used to solve the conflict, This method depends mainly on the experience of the decision-maker in the development of weights according to the importance of each objective and this will be explained in the next paragraph.

\section{9-4 Build a weighted mathematical model to solve the multi-objective of (TSP)}

The weighted mathematical model of the multi-objective of (TSP) can be represented as follows:

$$
\begin{aligned}
& \min F_{W}=\sum_{k=1}^{3} \omega_{k} f_{k}(\mathrm{x}) \\
& =\omega_{1} f_{1}(\mathrm{x})+\omega_{2} f_{2}(\mathrm{x})+\omega_{3} f_{3}(\mathrm{x})
\end{aligned}
$$

Subject To:

The same constraints $(6,7$ and 8$)$

as are mentioned above

The model is solved as follows:

Let us consider that the weights given by the decision maker are $\left(W=\left\{\omega_{1}=0.3, \omega_{2}=0.5, \omega_{3}=0.2\right\}\right)$, the total of these weights is $\left\{\sum_{k=1}^{3} \omega_{k}=1\right\}$

The weights given are the product of the decision maker's experience. In his view, the cost and time are dependent on the distance. Whenever the distance low, result the cost and time are low, vice versa, so after substituted the weights in the model (9), we get the following new model:

$\min F_{W=\{0.3,0.5,0.2\}}=$ $0.3 f_{1}(\mathrm{x})+0.5 f_{2}(\mathrm{x})+0.2 f_{3}(\mathrm{x})$

Subject To:

The same constraints $(6,7$ and 8$)$ as are mentioned above

After performing the mathematical operations by multiply the weights by the objectives and then collecting the objectives to be a single objective, i.e. converting the problem multi-objective to the problem of oneobjective based on the following tables: 


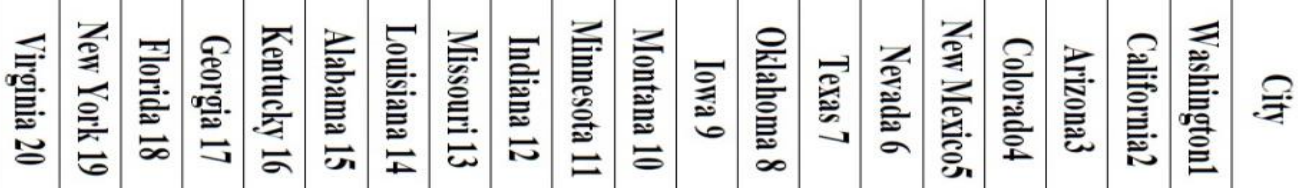

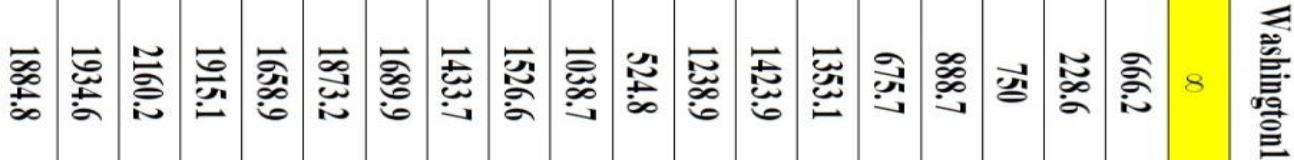

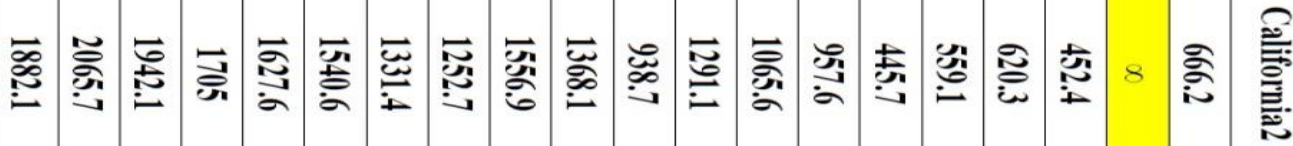

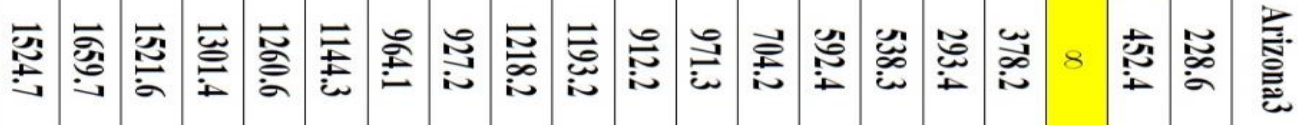

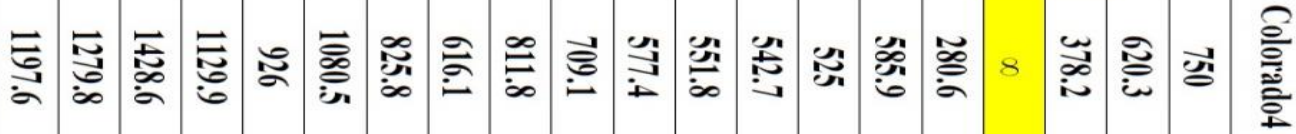

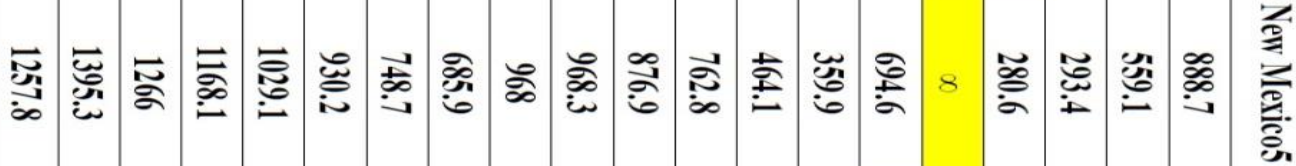

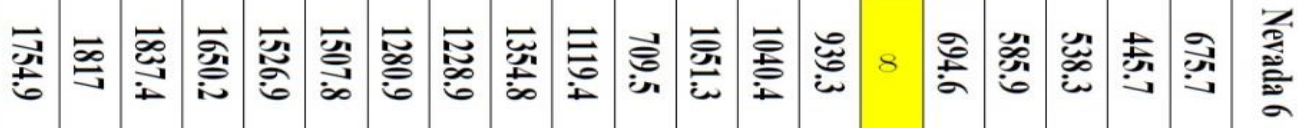

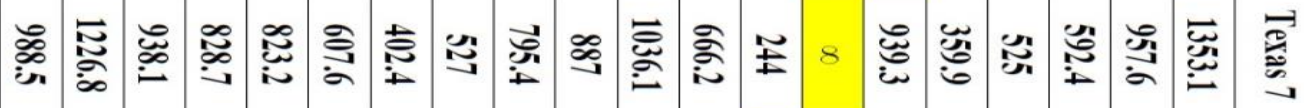

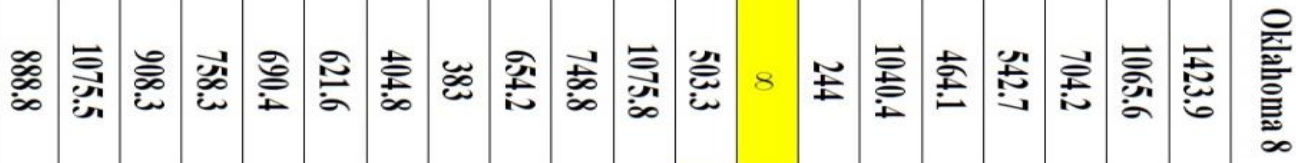

$\stackrel{8}{8}$

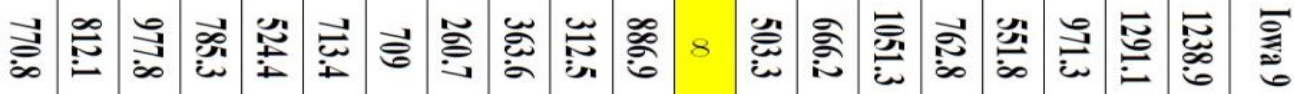

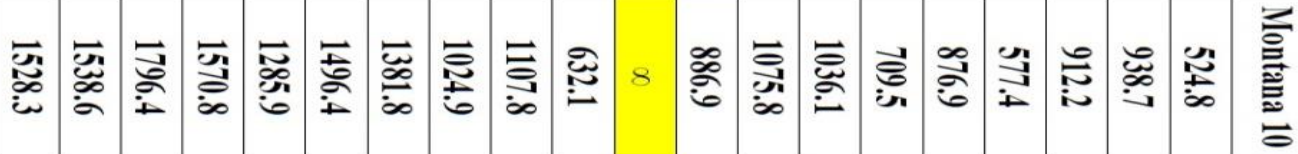




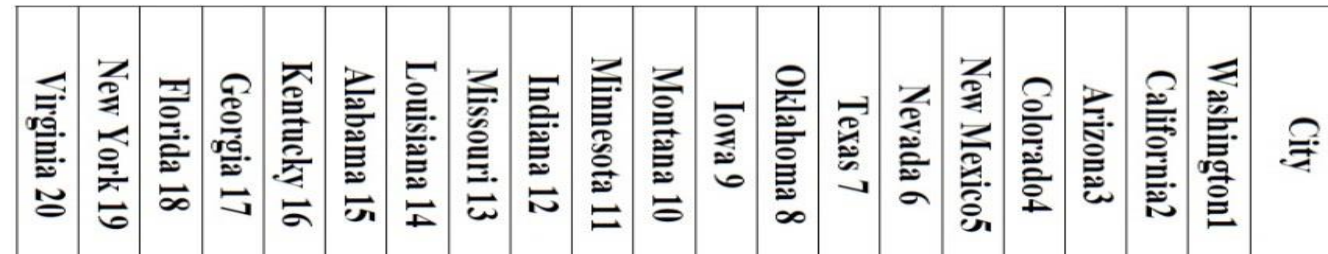

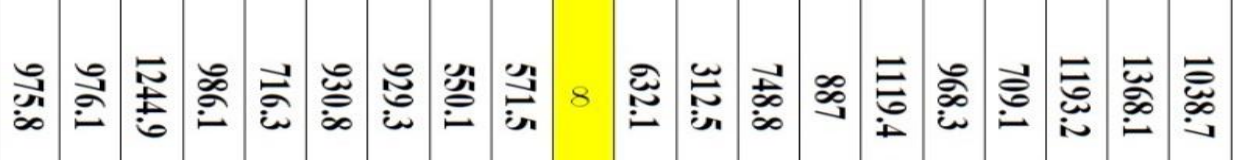

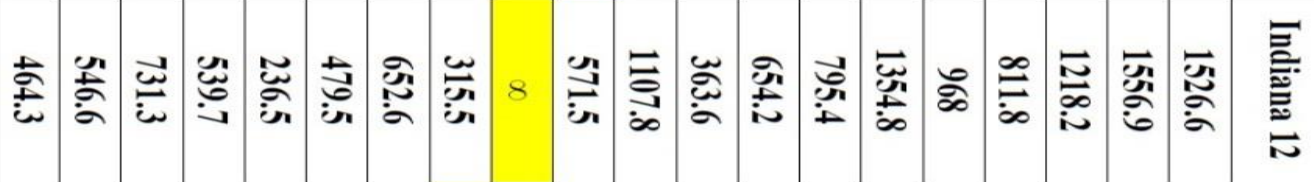

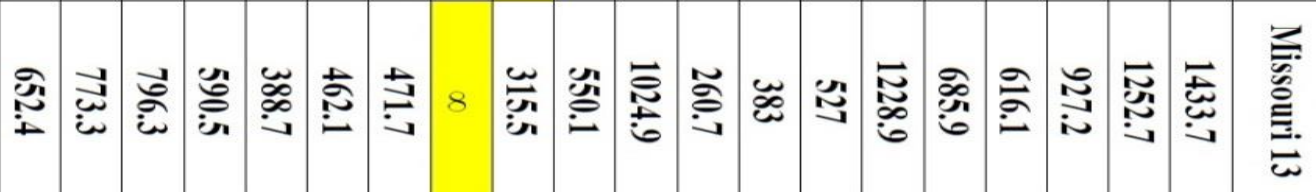

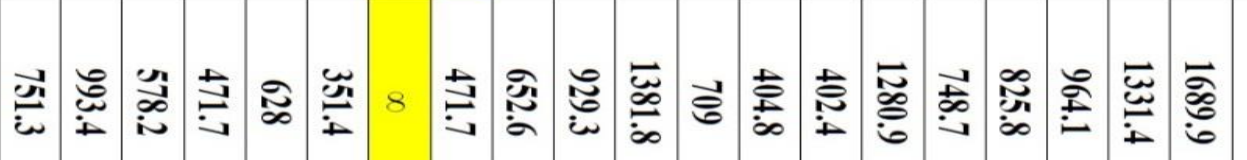

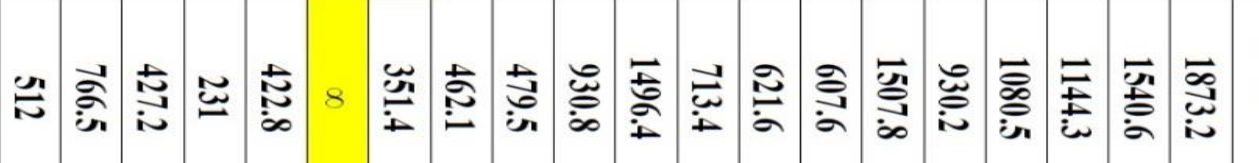

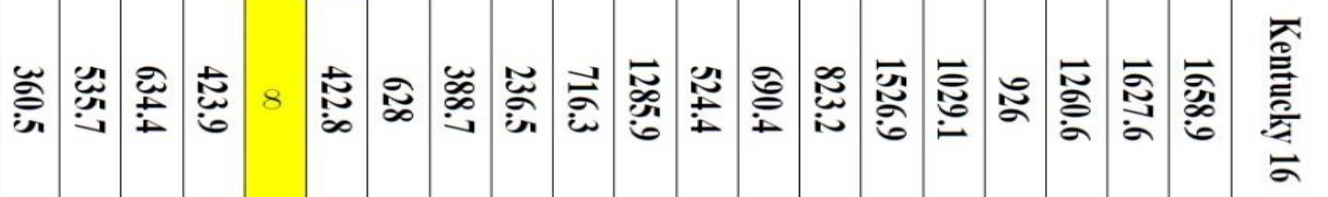

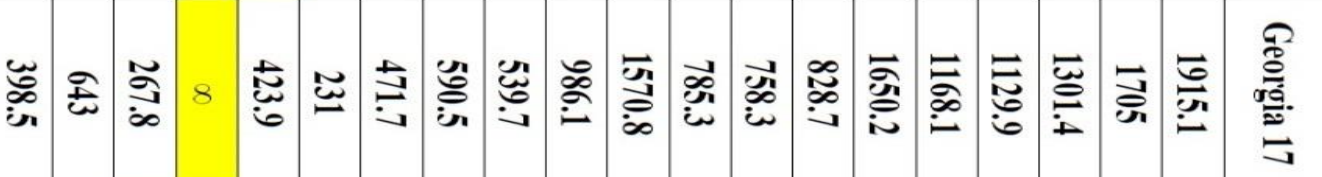

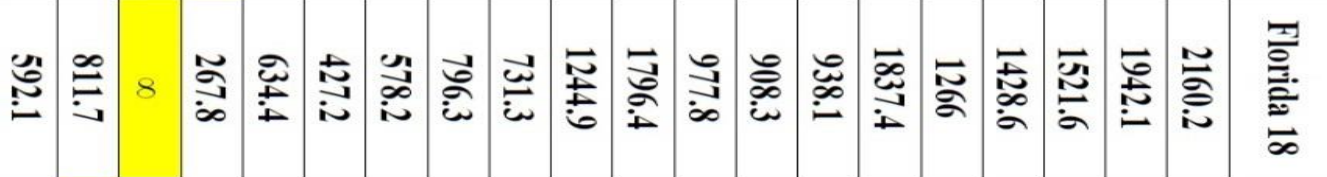

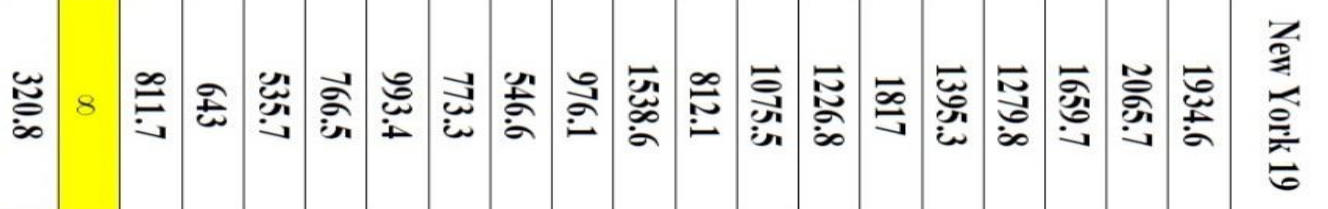

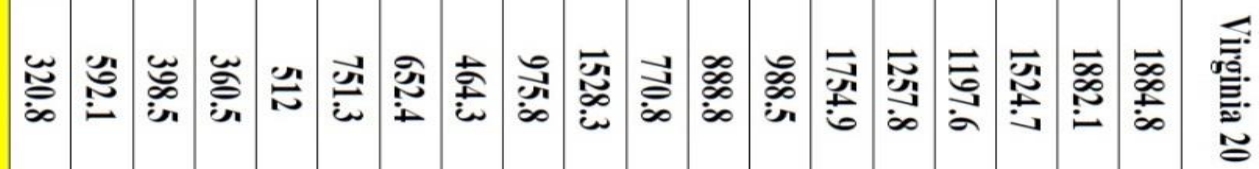


By the Tables (2-A and 2-B) above, the weighted objective function is as follows:

Min $F_{W}=666.2 x_{1,2}+228.6 x_{1,3}+750 x_{1,4}+888.7 x_{1,5}$

$+1238.9 x_{1,9}+675.7 x_{1,6}+1353.1 x_{1,7}+1423.9 x_{1,8}$

$+524.8 x_{1,10}+1038.7 x_{1,11}+1526.6 x_{1,12}+1433.7 x_{1,13}$

$+1689.9 x_{1,14}+1873.2 x_{1,15}+1658.9 x_{1,16}+1915.1 x_{1,17}$

$+2160.2 x_{1,18}+1934.6 x_{1,19}+1884.8 x_{1,20}+\ldots+1884.8 x_{20,1}$

$+1882.1 x_{20,2}+1524.7 x_{20,3}+1197.6 x_{20,4}+1257.8 x_{20,5}$

$+1754.9 x_{20,6}+988.5 x_{20,7}+888.8 x_{20,8}+770.8 x_{20,9}$

$+1528.3 x_{20,10}+975.8 x_{20,11}+464.3 x_{20,12}+652.4 x_{20,13}$

$+751.3 x_{20,14}+512 x_{20,15}+360.5 x_{20,16}+398.5 x_{20,17}$

$+592.1 x_{20,18}+320.8 x_{20,19}$
Subject To: The same constraints 6,7 and 8 as are mentioned above

\section{9-5 Solution for Minimization (Multi- objective Traveling Salesman Problem)}

In this section, the model (11) will be solved to obtain the optimal solution using the three methods (branch and bound, nearest neighbor and two-way exchange improvement heuristic) to solve the problem of (TSP), as well as a comparison among the optimal results to be obtained in the following manner, all results depended on the package program WINQSB [5]:

Table (3)

Represents the optimal solution by using $B$ and $B$.

\begin{tabular}{||c|c||c|c|c||c|c|c||}
\hline City & From Node & Connect To & Arc value & City & From Node & Connect To & Arc value \\
\hline \hline 1 & Node1 & Node2 & 666.2 & 11 & Node16 & Node13 & 388.7 \\
\hline 2 & Node2 & Node6 & 445.7 & 12 & Node13 & Node15 & 462.1 \\
\hline 3 & Node6 & Node4 & 585.9 & 13 & Node15 & Node17 & 231 \\
\hline 4 & Node4 & Node10 & 577.4 & 14 & Node17 & Node18 & 267.8 \\
\hline 5 & Node10 & Node11 & 632.1 & 15 & Node18 & Node14 & 578.2 \\
\hline 6 & Node11 & Node9 & 312.5 & 16 & Node14 & Node8 & 404.8 \\
\hline 7 & Node9 & Node12 & 363.6 & 17 & Node8 & Node7 & 244 \\
\hline 8 & Node12 & Node19 & 546.6 & 18 & Node7 & Node5 & 359.9 \\
\hline 9 & Node19 & Node20 & 320.8 & 19 & Node5 & Node3 & 293.4 \\
\hline \hline 10 & Node20 & Node16 & 360.5 & 20 & Node3 & Node1 & 228.6 \\
\hline \hline & Total & Minimal & $F_{W}$ & $=$ & $8,269.80$ & & \\
\hline & (Result & from & Branch & and & Bound & Method) & \\
\hline
\end{tabular}

Table (4)

Represents the optimal solution by using nearest neighbor algorithm.

\begin{tabular}{|c|c||c|c|c||c|c||c|}
\hline City & From Node & Connect To & Arc value & City & From Node & Connect To & Arc value \\
\hline \hline 1 & Node1 & Node3 & 228.6 & 11 & Node16 & Node20 & 360.5 \\
\hline 2 & Node3 & Node5 & 293.4 & 12 & Node20 & Node19 & 320.8 \\
\hline 3 & Node5 & Node4 & 280.6 & 13 & Node19 & Node17 & 643 \\
\hline 4 & Node4 & Node7 & 525 & 14 & Node17 & Node15 & 231 \\
\hline 5 & Node7 & Node8 & 244 & 15 & Node15 & Node14 & 351.4 \\
\hline 6 & Node8 & Node13 & 383 & 16 & Node14 & Node18 & 578.2 \\
\hline 7 & Node13 & Node9 & 260.7 & 17 & Node18 & Node10 & 1796.4 \\
\hline \hline 8 & Node9 & Node11 & 312.5 & 18 & Node10 & Node6 & 709.5 \\
\hline 9 & Node11 & Node12 & 571.5 & 19 & Node6 & Node2 & 445.7 \\
\hline 10 & Node12 & Node16 & 236.5 & 20 & Node2 & Node1 & 666.2 \\
\hline \hline & Total & Minimal & $F_{W}$ & $=$ & $9,438.50$ & & \\
\hline \hline & (Result & from & Nearest & Neighbor & Heuristic) & & \\
\hline
\end{tabular}


Table (5)

Represents the optimal solution by using Two-way exchange improvement heuristic.

\begin{tabular}{|c|c|c||c|c||c|c|c||}
\hline City & From Node & Connect To & Arc value & City & From Node & Connect To & Arc value \\
\hline \hline 1 & Node16 & Node12 & 236.5 & 11 & Node3 & Node5 & 293.4 \\
\hline 2 & Node12 & Node13 & 315.5 & 12 & Node5 & Node7 & 359.9 \\
\hline 3 & Node13 & Node9 & 260.7 & 13 & Node7 & Node8 & 244 \\
\hline 4 & Node9 & Node11 & 312.5 & 14 & Node8 & Node14 & 404.8 \\
\hline 5 & Node11 & Node10 & 632.1 & 15 & Node14 & Node15 & 351.4 \\
\hline 6 & Node10 & Node4 & 577.4 & 16 & Node15 & Node17 & 231 \\
\hline \hline 7 & Node4 & Node6 & 585.9 & 17 & Node17 & Node18 & 267.8 \\
\hline 8 & Node6 & Node2 & 445.7 & 18 & Node18 & Node20 & 592.1 \\
\hline \hline 9 & Node2 & Node1 & 666.2 & 19 & Node20 & Node19 & 320.8 \\
\hline 10 & Node1 & Node3 & 228.6 & 20 & Node19 & Node16 & 535.7 \\
\hline \hline & Total & Minimal & $F_{W}$ & $=$ & $7,862.00$ & & \\
\hline \hline & (Result & from & Two-way & Exchange & Improvement & Heuristic) & \\
\hline
\end{tabular}

After finding the optimal solutions above, a table will be made to compare the optimal solutions after substation the optimal binary decision variables in the three objective functions (cost, distance and time) as shown in Table (6) below.

Table (6)

Represents a comparison of optimal solutions with the given weights.

\begin{tabular}{|c|c|c|c|c|}
\hline Index & $\begin{array}{l}\text { Methods } \\
\text { (TSP) }\end{array}$ & $\begin{array}{c}\text { Objective fn1. } \\
(\text { cost })\end{array}$ & $\begin{array}{c}\text { Objective fn2. } \\
\text { (distance) }\end{array}$ & $\begin{array}{c}\text { Objective fn3. } \\
\text { (time) }\end{array}$ \\
\hline 1 & Branch \& Bound & 3688 & 10694 & 9082 \\
\hline 2 & Nearest neighbor & 4134 & 12299 & 10244 \\
\hline 3 & $\begin{array}{c}\text { Two-way exchange } \\
\text { improvement }\end{array}$ & 3817 & 10009 & 8562 \\
\hline \multicolumn{2}{|r|}{ Weight } & 0.3 & 0.5 & 0.2 \\
\hline
\end{tabular}

The objectives as important in terms of the weighted preference of the decision maker can be summarized as follows:

The highest weight $(0.5)$ is for distance that is the second objective, heuristic algorithm gave the maximum reduction.

The middle weight $(0.3)$ is for cost that is the first objective; $\mathrm{B} \& \mathrm{~B}$ algorithm gave the

The lowest weight (0.2) is for time that is the third objective, heuristic algorithm gave the maximum reduction.

Since the decision-maker is looking for reduce the distance to adopt the time and cost, The Heuristic algorithm is the best solution for the problem and the optimal path is as shown in Table (10). 
Table (7)

Represents the optimal path (rout) by Heuristic algorithm.

\begin{tabular}{|c|c|c|c|c|c|}
\hline Index & City (From - To) & Optimal Route & Cost $1 \$$ & Distancelmile & Timelminute \\
\hline 1 & Washington - Arizona & Start Travel (1) - r & 332 & 144 & 285 \\
\hline 2 & Arizona - New Mexico & $r-0$ & 227 & 433 & 44 \\
\hline 3 & New Mexico - Texas & $0-V$ & 183 & 450 & 400 \\
\hline 4 & Texas - Oklahoma & $v-\Lambda$ & 45 & 341 & 300 \\
\hline 5 & OKlahoma - Louisiana & $\wedge-14$ & 286 & 466 & 430 \\
\hline 6 & Louisiana - Alabama & $14-15$ & 278 & 388 & 370 \\
\hline 7 & Alabama - Georgia & $15-17$ & 135 & 277 & 260 \\
\hline 8 & Georgia - Florida & $17-18$ & 56 & 366 & 340 \\
\hline 9 & Florida - Virginia & $18-20$ & 172 & 805 & 690 \\
\hline 10 & Virginia - New York & $20-19$ & 156 & 388 & 400 \\
\hline 11 & New York - Kentucky & $19-16$ & 219 & 700 & 600 \\
\hline 12 & Kentucky - Indiana & $16-12$ & 250 & 243 & 200 \\
\hline 13 & Indiana - Missouri & $12-13$ & 190 & 389 & 320 \\
\hline 14 & Missouri - Iowa & $13-9$ & 114 & 325 & 320 \\
\hline 15 & Iowa - Minnesota & $9-11$ & 135 & 404 & 350 \\
\hline 16 & Minnesota - Montana & $11-10$ & 282 & 799 & 740 \\
\hline 17 & Montana - Colorado & $10-4$ & 158 & 780 & 700 \\
\hline 18 & Colorado - Nevada & $4-6$ & 48 & 803 & 850 \\
\hline 19 & Nevada - California & $6-2$ & 82 & 555 & 718 \\
\hline 20 & $\begin{array}{l}\text { California - } \\
\text { Washington }\end{array}$ & $2-$ End Travel (1) & 469 & 953 & 245 \\
\hline \multicolumn{3}{|c|}{ Total cost, distance and time } & 3817 & 10009 & 8562 \\
\hline
\end{tabular}

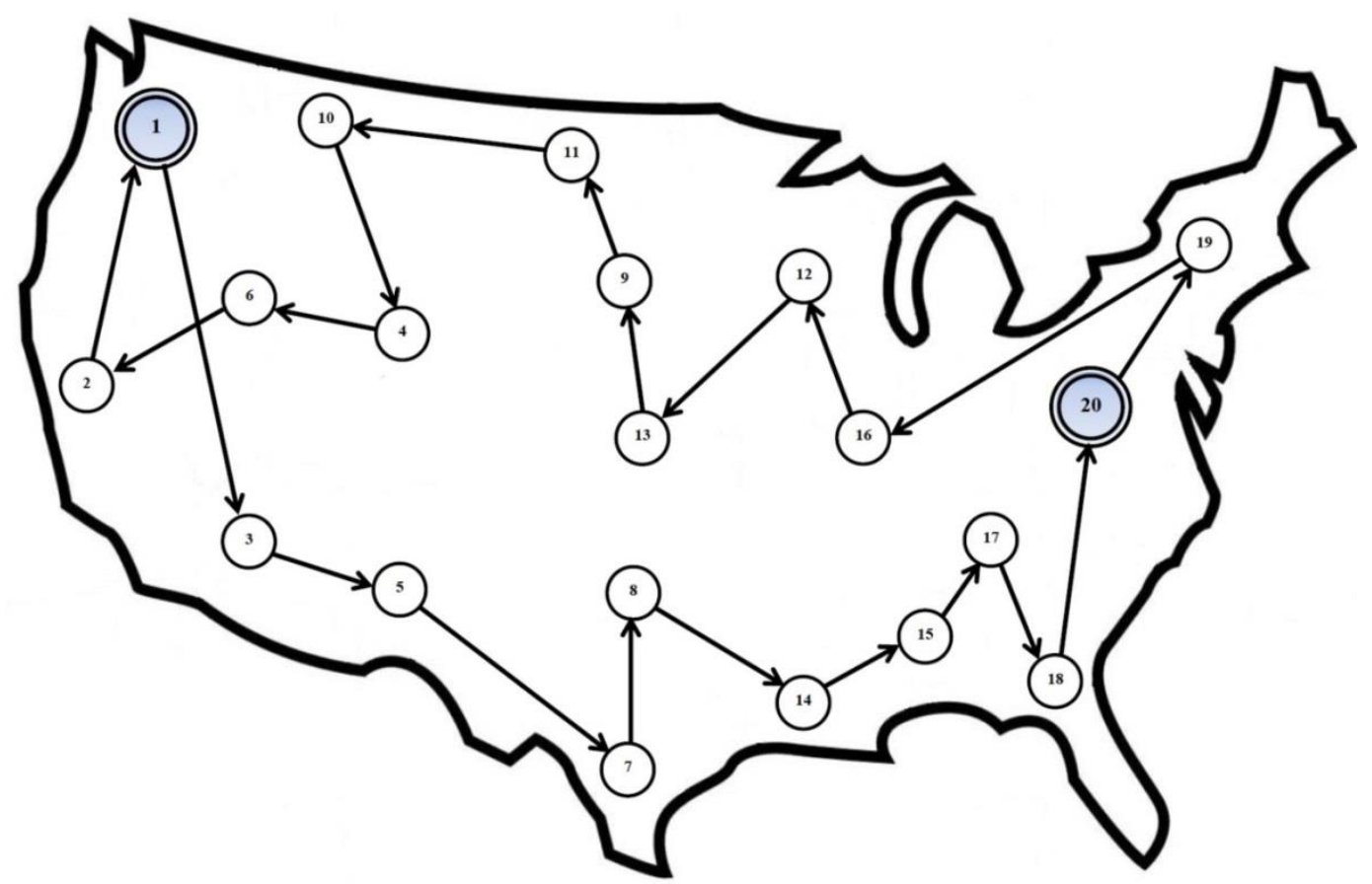

Fig.(1): Illustrates the optimal rout. 


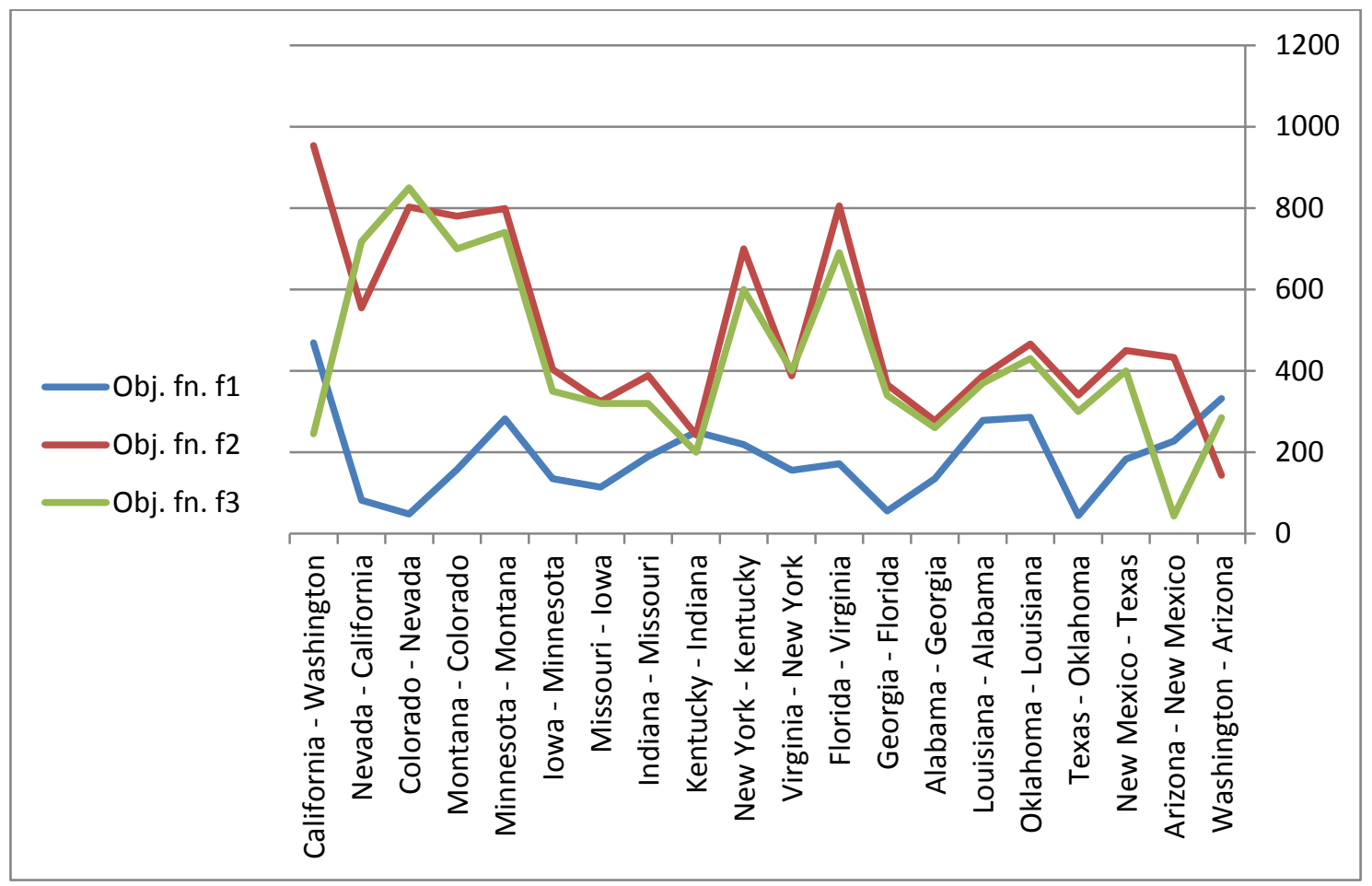

\section{Optimal Route}

Fig.(2): Chart illustrate optimal solution with objective functions $f_{1}, f_{2}, f_{3}$.

\section{Conclusions}

The weighted model demonstrated the effectiveness and flexibility to solve real problems of multi- objective (TSP), where it can be said that it is impossible to solve this problem without resorting to multipleobjective mathematical models, In other words, the number of possible rout for the 20 US states is $\{(n-1) !=19 !=121645100408832000\}$, to find the optimal routs among these routs it takes very long time and a lot of effort, here stand out importance of two-way exchange improvement heuristic algorithm, where this rout is satisfactory to the decision maker in terms of cost, distance and time.

\section{References}

[1] Ahmed M., "The use of the branch and bound algorithm and the genetic algorithm to solve travelling salesman problem", Journal of Statistical Sciences, 21(3), 69-96, 2012.

[2] Ibrahim M. S., Brraq S. K. "Use the optimum design to make the optimum decision when there are multiple objective", Al-Kout Journal of Economic and Administrative Sciences 26(2),22-25, 2017.
[3] Donald Davendra, "travelling salesman problems, theory and applications" ,intech,238-244, 2010.

[4] Tolga B., "The multiple traveling salesman problems: an overview of formulations and solution procedures", Omega, 34 (3), 209 219, 2006.

[5] Yih-Long C., "Win-QSB", (Published by Jon Willey and Sons), first edition, 2001.

[6] Moustaph D., M. Karwan H., "Linear programming formulations of the travelling salesman and other hard combinatorial optimization problems", world scientific publishing co, 22-25, 2015.

[7] Carlos Henggeler Antunes, Maria Joao Alves, and Joao Climaco "Multi-objective Linear and Integer Programming", Springer International Publishing Switzerland, 28-29, 2016.

[8] Sunarin C. and Ornurai S., "Application of AHP for Traveling Salesman Problem", Proceedings of the International Conference on Computer and Industrial Management ICIM, October 29-30, 2005, Bangkok, Thailand.

[9] https://www.google.com/maps/dir\& https://www.google.com/ flights/dir). 